\title{
An experimental communication architecture for monitoring and control of sub-grids
}

DOI:

10.5220/0003948600670072

Link to publication record in Manchester Research Explorer

\section{Citation for published version (APA):}

Pourmirza, Z., \& Brooke, J. (2012). An experimental communication architecture for monitoring and control of subgrids. In SMARTGREENS 2012 - Proceedings of the 1st International Conference on Smart Grids and Green IT Systems/SMARTGREENS - Proc. Int. Conf. Smart Grids Green IT Syst. (pp. 67-72). Science and Technology Publications Lda. https://doi.org/10.5220/0003948600670072

\section{Published in:}

SMARTGREENS 2012 - Proceedings of the 1st International Conference on Smart Grids and Green IT Systems|SMARTGREENS - Proc. Int. Conf. Smart Grids Green IT Syst.

\section{Citing this paper}

Please note that where the full-text provided on Manchester Research Explorer is the Author Accepted Manuscript or Proof version this may differ from the final Published version. If citing, it is advised that you check and use the publisher's definitive version.

\section{General rights}

Copyright and moral rights for the publications made accessible in the Research Explorer are retained by the authors and/or other copyright owners and it is a condition of accessing publications that users recognise and abide by the legal requirements associated with these rights.

\section{Takedown policy}

If you believe that this document breaches copyright please refer to the University of Manchester's Takedown Procedures [http://man.ac.uk/04Y6Bo] or contact uml.scholarlycommunications@manchester.ac.uk providing relevant details, so we can investigate your claim.

\section{OPEN ACCESS}




\title{
AN EXPERIMENTAL COMMUNICATION ARCHITECTURE FOR MONITORING AND CONTROL OF SUB-GRIDS
}

\author{
Zoya Pourmirza and John Brooke \\ School of Computer Science, The University of Manchester, Oxford Street, M13 9PL, Manchester, U.K.
}

Keywords: Smart Grid, Real-time Data, Communication, Network Architecture, Wireless Sensor Network, Monitoring,
Energy Efficiency, Distributed Systems.

Abstract: The Smart Grid promises to provide better monitoring and control by incorporating the communication network over the power network. We investigate the monitoring and control of distribution sub-Grids, for example to a local area in a city. We propose a communication architecture to be deployed into a real local area of the sub-Grid to provide a test bed for supporting real-time data and predictive real-time system control. This is one of the main challenges of the Smart Grid. Our system design is aimed at investigating two key issues: firstly, energy constraints in wireless sensor networks and secondly, achieving an appropriate balance between central and distributed control of the sub-Grid. We propose an energy efficient distributed architecture, for control and communication and explain how it will be implemented in our experimental test bed. Also, TinyDB, which is a query processing system for sensor networks, has been extended to collect the real-time data from the environment, and make them accessible by the local control unit. Finally, a visualization tool has been developed to integrate the map view of the test bed, display the real-time data, and send an alert to the network operator for finer-grained control over the system.

\section{INTRODUCTION}

The Smart Grid is considered as an integration of a power network and a communication network. The communication network, responsible for providing communication between different sectors in a power network by using digital technology, has three tiers known as wide area network, neighbourhood area network, and home area network. It is proposed (Ericsson, 2010) that a communication system will be a critical tool for the operation and administrative purposes of the future power grid. In this paper we describe a communication network architecture for a neighbourhood area and describe how it is being implemented in a test bed of sensors installed in the $6.6 \mathrm{kV}$ sub-Grid on a University Campus. Very little is known about the monitoring and control on this sub-Grid level, however as the local distribution networks cease to be purely passive but can be controlled at multiple levels, such a communications architecture will be essential for efficient operation. Our work is motivated by the study of other networked systems such as the water distribution grids (Khan et al., 2010, Machell et al., 2010, Stoianov et al., 2007), which integrates sensing, computation and decision making.

Our system design is targeted towards the solution of two key problems. The first is the energy constraint problem of general Wireless Sensor Network (WSN), (Abbasi and Younis, 2007, Raghunathan et al., 2004). The second problem addresses the weaknesses of a centralized architecture where the entire system data are stored at a sub-Grid database, where controls are applied.

The paper is structured as follows: in Section 2 we explain the design of our architecture, in Section 3 we justify the choice of technologies used for architecture implementation, in section 4 we describe how we use simulation to explore different possibilities for deployment. In Section 5 we demonstrate the actual experimental test bed that will be used to examine the performance of the architecture. It Section 6 we summarise the current status of our work and present future plans.

\section{SUB-GRID COMMUNICATION ARCHITECTURE}

Given that the predictive real-time system control 
necessitates having real-time information, in this architecture real-time data are collected, analysed, converted to information and finally acted upon.

As shown in the figure below, we propose a mixture of peer-to-peer (P2P) and hierarchical architectures that integrates sensors and distributed computation units in a unified communications network. Notice that the Figure 1 shows only one local area in the sub-Grid, while there exists a number of local areas in the sub-Grid. This architecture contains three layers of sensors for measuring and collecting the data. Each layer of sensors is selected according to the data type they measure. The sensors in layer one and two are located in the street levels, communicating wirelessly. The sensing units in the layer three are located at the substation level using both wireless and wired communications. Next layer is the test and control unit which apply some control over the substation level. Then the database layer will store the aggregated data received from the three sensing layers and the test and control unit. In future we need to figure out how much and for how long we can store the data in a database, and how often we need to refresh them. Finally the top level of the architecture, showing the head of the local area, is called the local control unit. It will apply some control over the entire local area. The local control unit can access the sensing units directly in emergency situations or indirectly through the database layer in normal scenarios for applying some control and decision-making. Given that each local area is likely to take the optimal decisions for its region, which might not be the optimal solutions for the entire system, another layer of communication should be added over the local control unit, in order to make each local area aware of the state of the other local areas, and enable them coordinate their control decisions. Thus a publisher/subscriber system should be added on top of this architecture that will provide asynchronous and loosely coupled communication. Consequently, while taking decisions, each local area not only decides the optimal solution for its local context, but also takes decisions that might be the optimal solutions for the whole system.

The advantage of the proposed architecture over the traditional one is that it is able to avoid single point of failure, if one of the local areas goes down, the rest can function as normal in the sub-Grid. More importantly, energy efficiency could be achieved through this architecture. Given that energy for sending data is higher than energy for computing data (Heinzelman et al., 2000), by reducing the travelling distance and adding more computation to the system, we may achieve an energy efficient architecture.

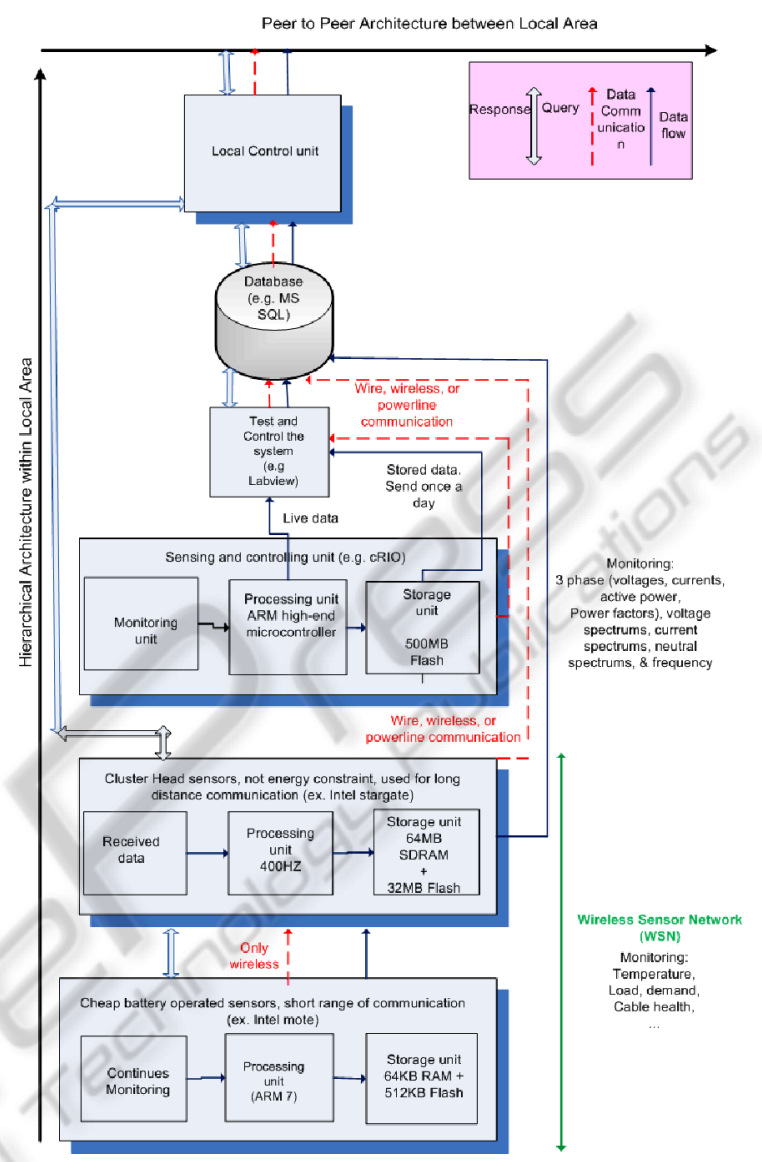

Figure 1: The proposed architecture.

For this architecture a simulation should be designed. It is supposed that initially data will be taken from the university campus to feed the simulation, and get the predictions of how the system should behave. Next, the theory (simulation) can be checked against the real world (experiments). Finally, the expert's feedback on this system will result in refining the architecture and designing an energy efficient communication network architecture suitable for Smart Grid applications.

\section{IMPLEMENTATION ISSUES}

In this architecture we provide a monitoring network that can be used by distributed control networks. The centralized control network is the norm in the electrical grid. Its advantage is that control can be optimized over the whole system. However, it has 
problems such as information flooding, single point of failure, less security and scalability. Migration from centralized control to distributed control network is also supported by Yang et al. (Yang et al., 2011) who believe that the current central control is no longer sufficient for the new power network with complex operational system and should be replaced by distributed control. In future by monitoring the response of the system to different control mechanisms, such as local control and optimized global control, we can propose an optimal control network structure.

Moreover, in this section we consider the choice of technologies used to implement the architecture and how we selected them in order to address the two problems mentioned in Section 1. We could eliminate the first problem, namely energy constraint by sending data from the sensors via existing electrical power lines. However information transmission over power lines has the following shortcomings (Galli et al., 2010): 1-Limited bandwidth, 2-Cable breakage will cause impaired connections, 3-Noisy channel, 4-Advanced noise filtering and error correction mechanism is required, 5-Communications between appliances of different vendors is a big challenge, 6 -Wire line technology needs a plug or socket to be connected to the device, which will result in nonflexible location of the device. Wireless technology is less reliable and secure but it offers low cost communication with various ranges of bandwidth. It provides communication to geographical areas that are not on the wired network. Nordell (2008) shows wireless can be used either for communications in the substation, or over longer path such as between substations or between enterprise and the substation. Moreover, wireless systems show resistance to electromagnetic interference of the system in high electric and magnetic fields in each substation. Thus for this reason we choose wireless technology as a means of communication.

Since we intend to integrate information gathering and control, an intelligent monitoring system should be implemented to keep track of collected data by sensor nodes. Then we need to feed the real-time data from the sensor network to the electrical network simulator. Having real-time data instead of historical data may help us to perform high-fidelity simulation that will result in more accurate predictions. In this system, unlike the other intelligent networks, we did not use SCADA (Supervisory Control and Data Acquisition). SCADA (Daneels and Salter, 1999) is only able to monitor the critical areas of a network; whereas, our architecture goes beyond that and monitor the entire network. Also SCADA system has slow data update rate, which makes it inefficient for Smart Grid applications (Qiu et al., 2011). Considering the weaknesses of the SCADA system, the lowest layer of this architecture designed with WSN.

For reasons of efficient utilisation of energy in collecting data from the WSN we use cluster-based communication. Each cluster has a cluster head, which can be a more a powerful sensor than the other sensors. The sensors will send their data to the cluster head wirelessly, and the cluster head is responsible for transmitting the data to the base station. A survey (Abbasi and Younis, 2007) on clustering algorithms for wireless sensor network, also confirm our assumption that the cluster based communication provide more efficiency and longer life time. The survey goes on to state that in addition to advantages of clustering such as network scalability and energy efficiency, clustering can localize the route set up within the clusters and consequently decrease the size of routing table stored at a single node. Moreover, the range of inter cluster interactions to the Cluster Head $(\mathrm{CH})$ is restricted, and redundant message exchange is prevented, which help in preserving communication bandwidth. In addition, since the network topology is stabilized with the help of clustering at sensor level, resulting in cuts on topology maintenance overhead. Also the sensors are only concerned about their connection to their $\mathrm{CH}$, and would not be affected by the modifications at inter-CH levels. On the other hand, based on application requirements, the events monitored can be either continual or intermittent. The continual monitoring ends up in generating considerable amount of traffic to be routed to the sink. In In-network data processing similar packets from different nodes can be aggregated in order to reduce the number of transmissions. Since communications are more energy consuming than computations, this technique can save substantial amount of energy. Also, given that the first level of sensors are using Bluetooth communication with short range of data transmission, a cluster head is provided to offer longer range of communication, and be able to transfer data from lowest level to the higher levels.

The cRIOs (compactRIO) used in the third layer of the hierarchy are control and acquisition units. These cRIOs can also measure various quantities and can be programmed and used in controlling and monitoring applications. The live data from cRIO will be sent to the next layer of the hierarchy, responsible for testing and controlling the substation 
level. This layer is using a graphical programming environment called LabVIEW for measuring, testing, and controlling the system. Additionally, the collected data from cRIOs will be transferred and saved in the database layer, which can be accessed by the local control unit. The local control unit, being the highest layer of the hierarchy can query the three sensing layers and access the real-time and historical data.

\section{SENSOR NETWORK SIMULATION}

In order to evaluate the optimal method of implementing the WSN, TOSSIM (Levis and Lee, 2003), an existing wireless sensor network simulator, has been selected which simulates the nodes running on TinyOS (Levis et al., 2005) operating system. Although TOSSIM is a more complex simulation compared to abstract simulations, but it enables users to take their implementation and run it on the actual mote. This feature motivates us to select TOSSIM over other existing simulations. Therefore, we are able to run and test our prototype implementation on the real physical environment as well as laboratory-based environment. Additionally, TinyDB (Madden et al., 2003) which is a query processing system has been extended to meet the needs of the Smart Grid. TinyDB is able to query the sensors and save the results in a database layer. The database can save real-time data received from sensor network plus historical data of the system. These data will then be delivered to a higher-level unit. One difference between TinyDB and traditional database is that when we post a query to the sensors with TinyDB installation we receive the real-time data instead of receiving archived data in reply. Given that sensors are battery-powered devices, we tend to reduce power consumption in the system as much as possible. A typical strategy of power-efficient innetwork processing algorithm minimizes the communication cost by applying filtering and aggregation inside the sensor network and provides routing of data from a physical environment to a pc. The reason why we select TinyDB over the other query processing systems is that, not only it does contain the same features of a conventional query processing (such as join, select, project, and aggregate information), but also it provides functionality for minimizing power consumption by using the acquisitional techniques. ACquisitional Query Processing (ACQP) (Madden et al., 2005) focuses on both conventional techniques as well as the new features of query processing. These new features focus on when, where, and how often data are sampled and send to a query processing operator. In contrast to conventional passive systems, power consumption can be minimized by focusing on the location and cost of data acquisition.

To extract the desired information from the electrical network TinyDB has been extended by adding the SmartGrid component for electrical network applications. This extension enables us to extract the environmental information about our prototype electrical network and feed them to another source of computation for the further analysis and gaining the predictive real-time system control. In future, this extension will be used to produce measured data from the real environment.

\section{VISUALISATION OF THE TEST BED}

In this section we will demonstrate the overlay of the electrical network information on the map view using Google Maps API (Application Programming Interface). This tool enables users to click on the desired section in the network and check the related data.

\subsection{Related Work}

The work in (Haines et al., 2009) consider the map based graphical user interface as a significant factor for water engineers which enable them to run 'Whatif' simulations and visualise the results of their simulations. They show how dynamic information and prediction of network state can be accessed using lightweight devices and visualised in user friendly Google Maps based web interfaces. Also, another study by (Stoianov et al., 2007) uses Google map to visualize the data related to water network. They enable their users to choose a sensor and extract the related data from the water grid. The Google map visualization techniques can be applied to the other networks such as electrical grids. They are used to retrieve the data and help the users to better understand the network condition. The figure 2 shows how data relating to the sensors can be overlaid on a GIS (Google Maps).

\subsection{Test Bed}

This real project is being implemented on the medium voltage power network of the University of 


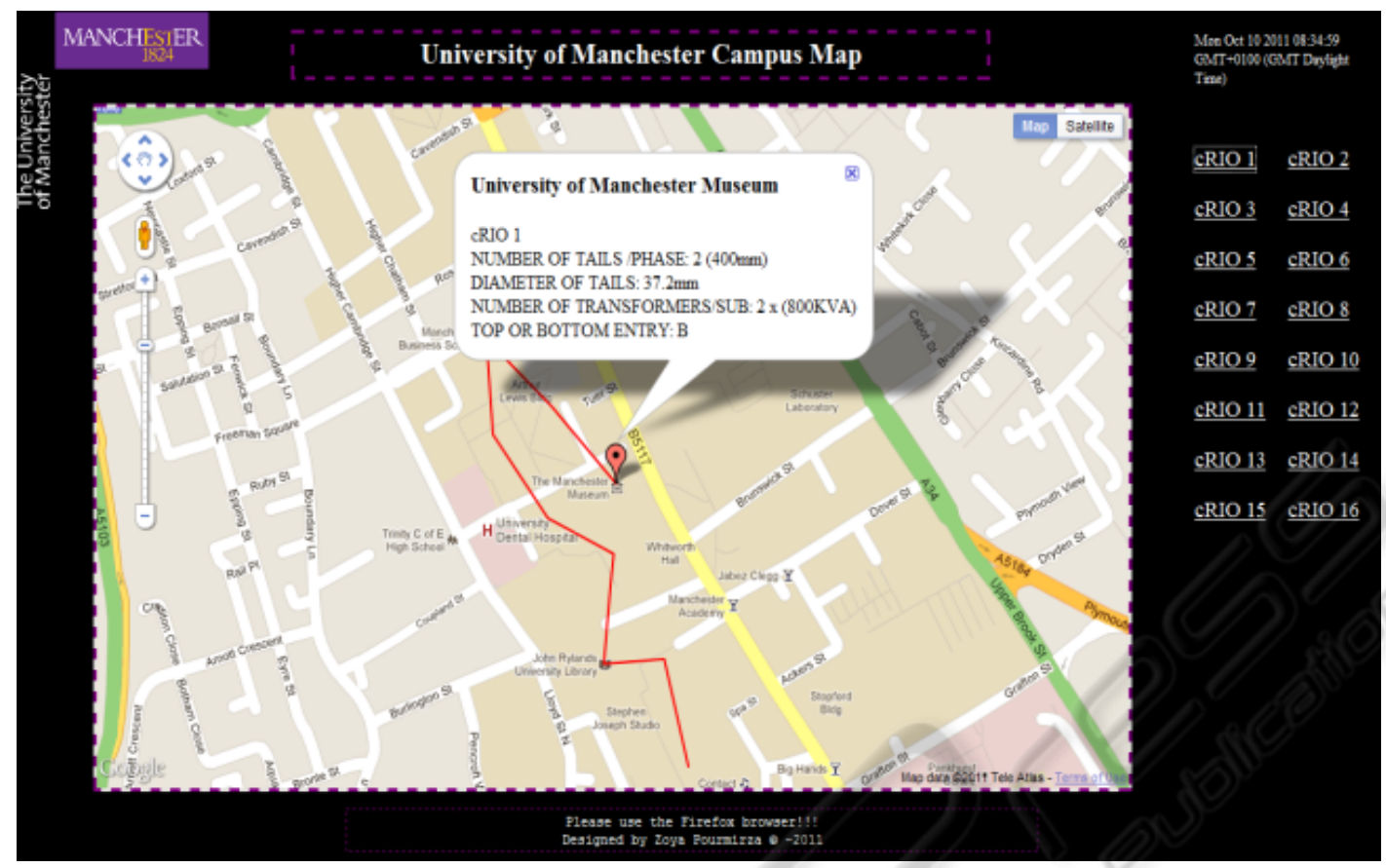

Figure 2: University of Manchester Campus.

Manchester campus. Also real equipment, real data, and professionals in the field are provided. We are now in the stage that this system is going live. Considering that currently there is no information available below $33 \mathrm{kV}$, our research is quite valuable since it will deliver the collected data to the experts and demonstrates what is happening in the lower voltage network that will consequently offer better control over the grid. This test bed only considers eleven $6.6 \mathrm{kV}$ substations that are equipped with sixteen monitoring systems called cRIO which collect the data from the network and process them. The substations with one transformer are equipped with one cRIO, whereas substations with two transformers are supplied with two cRIOs. These cRIOs are running at $4 \mathrm{~Hz}$, thus we are receiving four samples per second, measuring three phases voltages, currents, active powers, power factors, voltage's spectrums (eight channels for each phase) and current spectrums (eight channels) and frequency. Each substation can provide wireless and wired data communication to the data server.

In order to prevent data loss if the links go down, data should be stored locally in the cRIO memory and transferred once a day to the database layer. Subsequently, the live data will be sent, from cRIO to a pc running LabVIEW via TCP/IP or wireless communication to test and control the substation level. The live data will then be transferred and stored in the database layer. Given that better monitoring will result in more efficient control over the system, a graphical display of the network is provided for electrical engineers. It is helpful for them to utilize the visualization technology in order to track and discover the faulty part of the grid and take remedial actions. The figure 2 illustrates the University of Manchester campus map, where the red pin shows the first cRIO, and the rest of the cRIOs are link together with the red lines.

This tool enables the operators to observe the Google map view of the test bed and schematic view of the electrical network. The provided visualisation tool has some control functions such as panning and zooming. It also locates substations on the map according to their longitude and latitude, and demonstrates the related information. It is able to connect to the live database and data graph to display real-time data in order to help experts better comprehend what is happening in the local area network. If the measured data pass the defined threshold the tool will send an alert, so operators can recognize the fault very quickly and take corrective actions. In future we will add more functionality to the tool, and show the results of network simulations on the map. Briefly, it is assumed that the combination of the visualization technology and simulation technology allow operators to better predict the effect of course of actions by observing generated results of simulations on the map. 


\section{SUMMARY}

We have described how we have developed an architecture for monitoring and controlling the local area in the sub-Grids. We also describe how we intend to use simulation to tune the implementation of the architecture on a real test bed, namely a subGrid covering a large university campus. The particular interest of our work is the close connection between design and testing of the architecture and also that it will enable information to be gathered from the working of a real system operating a sub-Grid level where monitoring has not previously been deployed.

In future, this research will focus optimising the WSN topology and balancing between the cost of data transmission, data computation and data compression technique in order to bring more energy efficiency to the system. Also, the ever increasing volume of data gathered from the grid and the probability of data loss in the system, necessitate more investigations on data optimizing techniques in databases. Finally, further work needs to be done to establish publish-subscribe architecture to provide a P2P architecture between local areas.

\section{REFERENCES}

Abbasi, A. A. \& Younis, M. 2007. A survey on clustering algorithms for wireless sensor networks. Computer Communication, 30, 2826-2841.

Daneels, A. \& Salter, W. 1999. WHAT IS SCADA? In: Accelerator and Large Experimental Physics Control Systems 1999 Trieste, Italy. 339-343.

Ericsson, G. N. 2010. Cyber Security and Power System Communication-Essential Parts of a Smart Grid Infrastructure. IEEE TRANSACTIONS ON POWER DELIVERY, 25, 1501.

Galli, S., Scaglione, A. \& Wang, Z. 2010. Power Line Communications and the Smart Grid. In: First IEEE International Conference on Smart Grid Communications (SmartGridComm), 4-6 Oct. 303308.

Haines, R., Khan, K. \& Brooke, J. 2009. Bringing simulation to engineers in the field: a Web 2.0 approach. The Royal Society, 2635-2644.

Heinzelman, W. R., Chandrakasan, A. \& Balakrishnan, H. 2000. Energy-Efficient Communication Protocol for Wireless Microsensor Networks. In: Proceedings of the 33rd Hawaii International Conference on System Sciences-Volume 8, 820485: IEEE Computer Society, $1-10$.

Khan, K., Haines, R. \& Brooke, J. M. 2010. A Distributed Computing Architecture to Support Field Engineering in Networked Systems. In: Proceedings of the 2010 International Conference on Complex, Intelligent and Software Intensive Systems, 1796283: IEEE Computer Society, 54-61.

Levis, P. \& Lee, N. 2003. TOSSIM: A Simulator for TinyOS Networks. University of Berkeley. 115-148

Levis, P., Madden, S., Polastre, J., Szewczyk, R., Whitehouse, K., Woo, A., Gay, D., Hill, J., Welsh, M., Brewer, E. \& Culler, D. 2005. TinyOS: An Operating System for Sensor Networks Ambient Intelligence. Springer Berlin Heidelberg.

Machell, J., Mounce, S. R. \& Boxall, J. B. 2010. Online modelling of water distribution systems:a UK case study. Drinking Water Engineering and Science, 279_ 294.

Madden, S., Hellerstein, J. \& Hong, W. 2003. TinyDB: InNetwork Query Processing in TinyOS.

Madden, S. R., Franklin, M. J., Hellerstein, J. M. \& Hong, W. 2005. TinyDB: An Acquisitional Query Processing System for Sensor Networks. ACM Transactions on Database Systems, 30, 122-173.

Nordell, D. E. 2008. Communication systems for Distribution Automation. In: Transmission and Distribution Conference and xposition. IEEE/PES, 2124 April. 1-14.

Qiu, M., Gao, W., Chen, M., Niu, J. W. \& Zhang, L. 2011. Energy Efficient Security Algorithm for Power Grid Wide Area Monitoring System. IEEE TRANSACTIONS ON SMART GRID, 2, 715-723.

Raghunathan, V., Schurgers, C., Park, S. \& Srivastava, M. B. 2004. ENERGY EFFICIENT DESIGN OF WIRELESS SENSOR NODES. Wireless Sensor Networks. Kluwer Academic Publishers. 51-69

Stoianov, I., Nachman, L., Madden, S. \& Tokmouline, T. 2007. PIPENET a wireless sensor network for pipeline monitoring. In: Proceedings of the 6th international conference on Information processing in sensor networks, Cambridge, Massachusetts, USA. 1236396: ACM, 264-273.

Yang, Q., Barria, J. A. \& Green, T. C. 2011. Communication Infrastructures for Distributed Control of Power Distribution Networks. Industrial Informatics, IEEE Transactions on, 7, 316-327. 